\title{
Communication and psychosis: it's good to talk, but how?
}

Rose McCabe and Stefan Priebe

\begin{abstract}
Summary
communication between clinicians and patients is at the heart of psychiatric practice and particularly challenging with psychotic patients. It may influence patient outcome indirectly or be therapeutic in its own right. Appropriate conceptual models, evidence on effective interventions and specific training are required
\end{abstract}

to optimise communication in everyday routine practice.

\section{Declaration of interest}

None. This work was, in part, supported by the Medical Research Council (grant G0401323)
Rose McCabe (pictured) is a senior lecturer at Barts and the London School of Medicine and Dentistry. Her research focuses on linking treatment processes, particularly therapeutic relationships and interactions, with outcome. Stefan Priebe is Head of the Social and Community Psychiatry Research Unit at Barts and the London School of Medicine and Dentistry. His research addresses concepts, processes and outcomes in mental healthcare.

Clinicians communicate with patients. In psychiatry, this is arguably the main part of what they do in their daily practice. Yet, does it matter how they communicate? Both the General Medical Council and the Royal College of Psychiatrists highlight the role of good communication in achieving therapeutic relationships. Effective communication, and the related construct, the therapeutic relationship, may have an impact on patients engaging in treatment in the first place, following treatment suggestions, satisfaction, symptom severity, referral to other services and willingness to file lawsuits. ${ }^{1}$ It may even be therapeutic in its own right. The therapeutic relationship is negotiated and reflected in patient-clinician communication and appears to predict outcome in different samples and settings across mental healthcare. ${ }^{2}$ If communication may be influential in patient outcome, there is a challenge to understanding how these processes work in psychiatry. This may feel especially difficult when communicating with patients with psychosis whose contributions may appear to be inappropriate both in their content and placement in the interaction. The first step is good research.

\section{Studying communication}

Communication is difficult and cumbersome to study. A typical approach involves recording the interaction. Videotaping one session can be the minimum. This is easier in a clinic setting than in various community settings. Audiotaping alone is problematic given how much information is contained in non-verbal aspects such as posture and gaze. A long gap in a consultation has a different meaning if the clinician is writing notes in that gap or has eye contact with the patient and is not responding to a patient's question. Most methods involve transcription, ranging from basic (content only) to highly detailed transcripts (content plus intonation, pauses, overlap, gaze, etc.) followed by time-consuming and labour-intensive analysis; linking one-off consultations with long-term clinical outcome is inappropriate given the complexity of treatment processes. It is likely that a series of consultations need to be studied to establish factors that have an impact on clinical outcomes. Simpler methods may need to be developed to capture intermediary outcomes of communication so that they can be assessed in pragmatic studies with sufficiently large samples.

Setting aside the methodological problems, a key conceptual issue is that, even in the social sciences, there is no definitive model of 'good communication'. A focus of positive communication throughout healthcare is patient-centredness. One component is shared decision-making. People with schizophrenia have a slightly stronger preference for shared decision-making than primary care patients. ${ }^{3}$ Among those with schizophrenia, younger people and those with more negative views of medication want more participation. ${ }^{3}$

Some research has been carried out on shared decisionmaking in relation to antipsychotics. Seale et al audiotaped psychiatric consultations and interviewed psychiatrists about their negotiating styles. ${ }^{4}$ In interviews, psychiatrists were committed to achieving concordant relationships with patients although they felt there were obstacles particular to psychiatry, mainly if the patient was deemed too ill to make decisions and the patient's honesty about their medication use. Analysis of the consultations themselves showed how side-effects may remain unaddressed (by offering no response, changing the subject or disagreeing with the patient's interpretation of the experience) or be acknowledged through sympathetic and supportive listening.

\section{What should I say now?}

In a detailed study of how psychiatrists and patients communicate about psychotic symptoms in out-patient consultations, patients repeatedly attempted to raise the content and emotional consequences of their hallucinations and delusions. ${ }^{5}$ Psychiatrists frequently avoided engaging with these concerns, leaving both patients and doctors very uncomfortable. One patient asked 'Why don't people believe me when I say I'm God?' to which the doctor, after initial avoidance, replied 'What should I say now?' In 'normal' interaction, avoiding sensitive issues that might expose conflicts of opinion is typically a good strategy. This might also apply here where the clinician avoided a confrontation about beliefs on which agreement was unlikely to be reached. Yet, in interactions with patients with psychosis, initial avoidance by clinicians seems to lead to explicit confrontation and disagreement about the very reason the patient is there. With patients who are not well-engaged, this might lead to further disengagement during treatment.

Despite the fact that communication about psychotic symptoms is a frequent challenge and regarded as fascinating by 
many clinicians, there is little systematic, theoretically informed training on how clinicians should respond. Many state that the recommended approach is not to 'encourage' the patient to talk about their symptoms because it amounts to inadvertent collusion about the illness. Because the patient is uncertain about reality, the clinician might feel that they should be firmly rooted in reality and respond to the God question with 'because it is not true'.

There are alternative ways to respond. For instance, a clientcentred approach might respond to the emotional content of the patient's statement with 'You feel misunderstood and puzzled by it'. A cognitive approach might ask for evidence about the belief. One could take the patient's perspective with a response like 'Why should people believe you? They did not believe Jesus either'. Further responses are possible using other therapeutic approaches. Yet, most psychiatrists are not specifically trained in this nor is there much theoretical debate on such a core aspect of everyday communication with patients with psychosis.

Communication is not only technical. It also involves emotions, particularly when communicating about profoundly disturbing experiences. Jaspers ${ }^{6}$ discussed the challenge of communicating with another person whose experience is so remote from the 'normal' realm to render it 'non-understandable'. However, in order to establish 'non-understandability' the clinician first has to try to understand the patient's experiences, which requires communication about symptoms, emotions and their meaning for the patient. Clinicians themselves may need to be supported in their response to patients' disturbing experiences. ${ }^{7}$

Communication involves at least two people and so far we have considered only the role of the clinician. However, doctors and patients construct the interaction together so how are patients with psychosis communicating? It is clear that patients are representing concerns that have been discussed many times before. They raise the same issues time and time again, often expecting that the clinician will disagree. ${ }^{8}$ It may be important to understand if (and how) patients are breaching 'normal' communicative practices both for understanding the disorder and identifying appropriate ways to respond.

\section{Interventions to improve communication}

In medicine generally, alerting clinicians to the patient's concerns/ emotions and changing clinicians' beliefs about communication have led to communication change. However, interventions to improve communication, and in turn outcome, in psychosis are rare. A simple communication checklist completed by patients before seeing their clinician improved communication and resulted in treatment changes. ${ }^{9}$ An intervention structuring patient-keyworker communication elicited the patient's satisfaction with a range of life domains, their needs for care and wishes for different help. Patients receiving the intervention had a better quality of life, fewer needs for care and higher treatment satisfaction after 1 year. ${ }^{10}$ It remains unclear, however, whether the structuring, focusing on the patient's view, the forward-looking emphasis on treatment changes or a combination of these factors was crucial to the intervention's success. Finally, an intervention to increase shared decision-making with in-patients with schizophrenia did not take up more of the doctor's time, increased the uptake of psychoeducation and increased involvement in medical decisions. ${ }^{11}$ As in medicine generally, different approaches have been tried on a more or less ad hoc basis without explicit theoretical frameworks specifying key communication processes and the pathway through which they may influence health outcomes.

The current state of the art cannot begin to address the question 'Does one size fit all?' (which is unlikely). Different clinicians may have different communication styles and strengths which might have to be enhanced rather than eradicated. Also, a particular clinician's communicative style may suit one patient and not another. Future research might address matching the right patient with the right clinician to achieve the best possible communication.

\section{Concluding remarks}

If psychiatrists want to make better use of everyday communication as a core component of their trade, the ambition must be to develop better competence and skills to maximise its therapeutic effect, preferably based on sound conceptual models and evidence derived from them. Some of the required skills may be generic, whereas others are likely to be specific to communicating with patients with psychosis. Jaspers ${ }^{6}$ stated that the ultimate thing in the doctor-patient relationship is existential communication, which goes far beyond any therapy, that is, beyond anything that can be planned or methodically staged' (p. 798). Thus, not all aspects of how psychiatrists and patients communicate might be identifiable in research and teachable in the classroom or individual supervision. Yet, the challenge is to advance the state of the art to reveal as much as possible so that patients benefit from communication that is, either indirectly or directly, therapeutic. Clinicians may also benefit from enriching their therapeutic options and professional expertise.

\footnotetext{
Rose McCabe, PhD, and Stefan Priebe, FRCPsych, Unit for Social and Community Psychiatry, Barts, and the London School of Medicine and Dentistry, Queen Mary, University of London, UK

Correspondence: Dr Rose McCabe, Unit for Social and Community Psychiatry, Newham Centre for Mental Health, London E13 8SP, UK. Email: r.mccabe@qmul.ac.uk

First received 11 Dec 2007, final revision 11 Dec 2007, accepted 14 Feb 2008
}

\section{References}

1 Cruz M, Pincus HA. Research on the influence that communication in psychiatric encounters has on treatment. Psychiatr Serv 2002; 53: 1253-65.

2 Castonguay LG, Beutler LE. Principles of Therapeutic Change That Work. Oxford University Press, 2006.

3 Hamann J, Cohen R, Leucht S, Busch R, Kissling W. Do patients with schizophrenia wish to be involved in decisions about their medical treatment? Am J Psychiatry 2005; 162: 2382-4.

4 Seale $C$, Chaplin R, Lelliott P, Quirk A. Sharing decisions in consultations involving anti-psychotic medication. A qualitative study of psychiatrists' experiences. Soc Sci Med 2006; 62: 2861-73.

5 McCabe R, Heath C, Burns T, Priebe S. Engagement of patients with psychosis in the consultation: conversation analysis study. BMJ 2002; 325: 1148-51.

6 Jaspers K. General Psychopathology (trans. J Hoenig, M Hamilton). Manchester University Press, 1959

7 Hinshelwood RD. Suffering Insanity: Psychoanalytic Essays on Psychosis. Brunner Routledge, 2004.

8 McCabe R. Specifying interactional markers of schizophrenia in clinical consultations. In Against Theory of Mind (eds I. Leudar, A. Costall). Palgrave Macmillan, in press.

9 Van Os J, Altamura AC, Bobes J, Gerlach J, Hellewell JSE, Kasper S, Naber D, Robert P. Evaluation of the Two-Way Communication Checklist as a clinical intervention. Br J Psychiatry 2004; 184: 79-83.

10 Priebe S, McCabe R, Bullenkamp J, Hansson L, Lauber C, Martinez-Leal R, Rössler W, Salize H, Svensson B, Torres-Gonzales F, Van Den Brink R, Wiersma D, Wright DJ. Structured patient-clinician communication and 1 -year outcome in community mental healthcare. Cluster randomised controlled trial. Br J Psychiatry 2007; 191: 420-6.

11 Hamann J, Langer B, Winkler V, Busch R, Cohen R, Leucht S, Kissling W. Shared decision making for in-patients with schizophrenia. Acta Psychiatr Scand 2006; 114: 265-73. 